\title{
Higher harmonics in complex plasmas with alternating screening
}

\author{
Z. A. Moldabekov $\odot,{ }^{1,2, *}{ }^{*}$ Y. K. Aldakul $\odot,{ }^{3}$ N. K. Bastykova $\odot,{ }^{4}$ S. Sundar, ${ }^{5}$ and A. Cangi $\odot^{1,2}$ \\ ${ }^{1}$ Center for Advanced Systems Understanding (CASUS), D-02826 Görlitz, Germany \\ ${ }^{2}$ Helmholtz-Zentrum Dresden-Rossendorf (HZDR), D-01328 Dresden, Germany \\ ${ }^{3}$ Leibniz-Forschungsinstitut für Molekulare Pharmakologie (FMP), Robert-Rössle-Straße 10, 13125 Berlin, Germany \\ ${ }^{4}$ Al-Farabi Kazakh National University, 71 Al-Farabi street, 050040 Almaty, Kazakhstan \\ ${ }^{5}$ Department of Aerospace Engineering, Indian Institute of Technology Madras, Chennai - 600036, India
}

(Received 9 August 2021; accepted 23 November 2021; published 17 December 2021)

\begin{abstract}
We report how higher harmonics of collective excitations emerge in a two-dimensional layer of strongly correlated charged microparticles in a complex plasma with a periodically alternating screening. The simulation results of the radio frequency discharge and the charged microparticles are obtained using a highly accurate multiscale and multiphysics approach based on the particle-in-cell technique, Monte Carlo collision calculations, and molecular dynamics simulations. We also devise a simple phenomenological expression for the dispersion relation of higher harmonics. Furthermore, our analysis reveals that the periodically alternating screening causes a self-conjugate state with negative refraction. In doing so, we demonstrate how complex plasmas can serve as a test bed for studying the fundamental physics of a self-conjugate state in strongly correlated systems.
\end{abstract}

DOI: 10.1103/PhysRevResearch.3.043187

\section{INTRODUCTION}

Collective phenomena such as oscillations and waves in strongly correlated many-particle systems are of both fundamental and practical importance [1-4]. The interaction between particles in such systems significantly varies depending on the nature of interacting particles, external fields, and other system parameters. Many technological innovations are based on emerging collective phenomena in various types of many-particle systems such as in condensed matter [5,6], plasmas [7-10], and warm dense matter [11,12]. Arguably the most prominent example is superconductivity associated with the attraction between electrons mediated by phonons [13]. The lessons learned from this and many other examples underpin the insatiable interest in discovering novel collective excitations in correlated systems.

However, generating and observing collective phenomena is challenging. Therefore a number of laboratory model systems serve as a practical surrogate for studying highly correlated many-particle structures under controlled conditions. Model systems such as ultracold atoms provide insights into the inherent dynamics of purely quantum phenomena [14]. Likewise, complex (dusty) plasmas and colloidal suspensions are used in the context of classical strong-correlation phenomena [15]. More recently, ultracold dusty plasmas [16-18] have been utilized to study the effects that ultracold environments

\footnotetext{
*z.moldabekov@hzdr.de

Published by the American Physical Society under the terms of the Creative Commons Attribution 4.0 International license. Further distribution of this work must maintain attribution to the author(s) and the published article's title, journal citation, and DOI.
}

have on nano- and microparticle dynamics. These model systems probe otherwise inaccessible quantities and create states that are difficult to observe in nature.

Complex plasmas have also been successfully used as a laboratory model system for investigating nonideal twodimensional (2D) systems [19,20]. In experiments, charged microparticles are observed in terms of a few isolated dust grains or as an ensemble of grains interacting with each other. Under the influence of external electric and magnetic fields, they exhibit a myriad of physical phenomena. For example, studying complex plasmas has led to the discovery of various new waves in strongly correlated systems and enabled an understanding of both single-particle and collective oscillations $[3,4]$. Additionally, the physics of non-Hamiltonian and selforganizing open systems $[15,21]$ has been investigated with the aid of a 2D system of charged microparticles in plasmas.

In this paper, we extend the realm of complex plasma physics to the nonlinear regime of higher harmonics. We predict the generation of multiple higher harmonics in a $2 \mathrm{D}$ system of strongly correlated charged microparticles interacting via a screened pair potential with alternating screening length. We also obtain a simple phenomenological expression that accurately represents these higher harmonics and provides stability criteria. We exemplify all this for complex plasmas generated in capacitively coupled radio frequency (RF) argon discharges. Our results are based on highly accurate multiscale and multiphysics simulations. The RF discharge simulation is based on the combination of the selfconsistent particle-in-cell technique with the Monte Carlo method (PIC-MCC, where MCC refers to Monte Carlo collisions) [22]. The time evolution of the microparticles is obtained from molecular dynamics (MD) simulations that are driven using the numerical data generated from the PIC-MCC calculations. 
The dynamics of electrons, ions (atoms), and microparticles takes place on substantially different characteristic time scales due to their different masses. Numerical simulations of the dynamics in complex plasmas composed of these particles are therefore highly challenging. However, they become feasible under certain assumptions. Assuming that a micrometer-sized dust particle does not respond to the details of a rapidly changing spatial profile of the electron density and temperature, we employ a multiscale approach consisting of three levels: (i) PIC-MCC simulations of the discharge covering the time scale up to several hundred microseconds and capturing processes at the times scale of nanoseconds such as gas ionization; (ii) calculations of the complex plasma parameters such as the dust particle charge, levitation length, and screening length using data from PIC simulations; and (iii) MD simulations of the charged microparticles with a given pair-interaction potential on the time scale of seconds. This multiscale approach allows us to define realistic parameters for the observation of the higher harmonic excitations in dusty plasmas.

The paper is composed as follows: In Sec. II we elaborate on the simulation methods used to set up a system of strongly correlated charged microparticles in complex plasmas. Our results are then presented in Sec. III. We conclude the paper in Sec. IV by discussing the relevance of the obtained results in the context of their experimental realization and by providing an outlook on future applications. Further technical details of our simulation methods are provided in Appendix A.

\section{SIMULATION METHODS AND PLASMA PARAMETERS}

Our analysis relies on setting up a numerical workflow that provides sustained conditions for a 2D layer of dust particles interacting via a repulsive Debye potential with a screening length that alternates in time. We achieve this by modulating the external RF voltage in combination with the external dc field. We implement a numerical workflow that is realizable in experiment by applying PIC-MCC together with the theory of dust particle-plasma interaction. Both methods have already been used to describe the RF discharge [23,24] in a complex plasma with high precision. Thus our numerical setup provides predictive capabilities.

The RF discharge, which is central to our workflow, is modeled as follows. We modify the standard external field excitation of a discharge with a harmonic RF voltage by imposing a modulation and an additional dc voltage. The parameters for the modulation and dc voltage are tuned in such a way that the charge over a dust particle remains almost unchanged, $\partial Z_{d} / \partial t \simeq 0$ (with a variation of about $2 \%$ ), compared with a standard harmonic RF voltage. By switching between modified and standard regimes, we realize a stable 2D layer of dust particles with a constant levitation height but with an alternating screening length of the argon plasma. Notably, the frequency of change in the screening length, $\Omega$, is defined as an external parameter. Here, we consider $\Omega>\omega_{d}$, where $\omega_{d}$ is the plasma frequency of charged dust particles. This keeps the discharge stable between the regimes where either a standard harmonic RF voltage or an alternating voltage with an additional dc component is applied. This is due to the

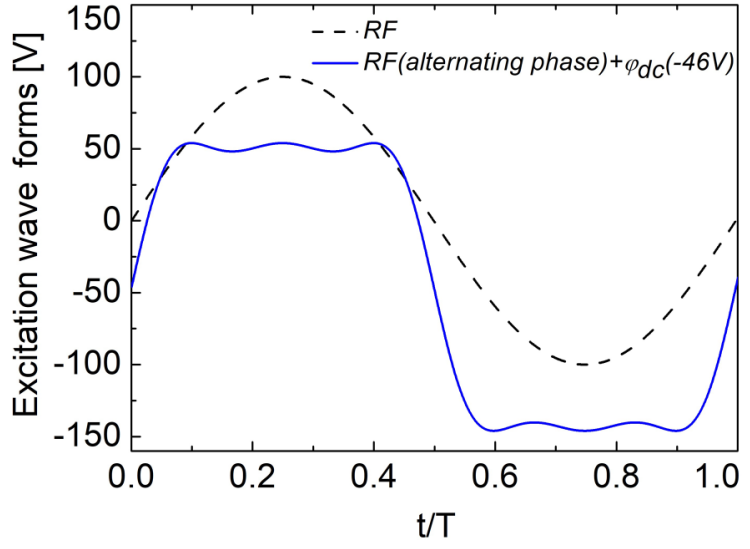

FIG. 1. Voltage signals which are applied to the electrodes where time is given in units of $T=2 \pi / f_{\mathrm{RF}}$.

fact that the characteristic time of the discharge stabilization is much shorter than the dust particle plasma period.

The RF discharge is ignited between two flat, horizontally opposing aluminum electrodes at a distance of $L=55 \mathrm{~mm}$ from each other and with a power supply operating at a frequency $f_{\mathrm{RF}}=13.56 \mathrm{MHz}$. The electrodes are located inside a glass cylinder. An argon gas is considered at a pressure $p=1.8 \mathrm{~Pa}$ and a gas temperature $T_{\mathrm{g}}=350 \mathrm{~K}$. The following boundary conditions are applied on the electrodes: One is supplied with a potential $\phi(t)$, and the other is grounded.

A constant levitation height of dust particles with an alternating plasma screening length is realized by switching between the following types of voltage signals: (1) the standard harmonic RF voltage, $\phi(t)=\phi_{0} \sin \left[2 \pi f_{\mathrm{RF}} t\right]$; and (2) an alternating voltage with an additional $\mathrm{dc}$ bias, $\phi(t)=\phi_{0} \sin \left\{2 \pi f_{\mathrm{RF}}(t)+\sin \left[2 \pi\left(2 f_{\mathrm{RF}} t\right)\right]\right\}+\phi_{\mathrm{dc}}$, where $\phi_{\mathrm{dc}}=-46 \mathrm{~V}$. The amplitude is set to $\phi_{0}=100 \mathrm{~V}$. These excitation wave forms are shown in Fig. 1. We note that the value of $\phi_{\mathrm{dc}}$ is defined by the condition to keep the levitation position of dust particles nearly constant when an alternating voltage is applied. We identify the actual value $\phi_{\mathrm{dc}}=-46 \mathrm{~V}$ by performing a series of test PIC-MCC simulations and corresponding calculations of the levitation position.

After performing PIC-MCC simulations, the equilibrium levitation position of the dust particle is derived from a forcebalance equation considering the electrostatic force, gravity force, and ion-drag force. We consider a spherical melamineformaldehyde particle with a radius of $2.19 \mu \mathrm{m}$ and a mass density of $1.51 \mathrm{~g} / \mathrm{cm}^{3}$. The Debye screening length $\lambda_{D}$ and the dust particle charge $Z_{d}$ are computed using argon plasma parameters at the equilibrium levitation position. Accordingly, we found that (a) when the harmonic RF excitation is applied, $\lambda_{D}=6.153 \times 10^{-5} \mathrm{~m}$ and $Z_{d}=-13990$; and (b) when the alternating-phase excitation with dc bias is considered, $\lambda_{D}=$ $4.361 \times 10^{-5} \mathrm{~m}$ and $Z_{d}=-13692$. Note that the screening length was computed using the average of plasma parameters over the period of the fundamental frequency $(13.56 \mathrm{MHz})$. Further details of the PIC-MCC simulations and of the solution of the force-balance equation are provided in Appendix A.

In addition to the screening length modulation frequency $\Omega$, there are two other free parameters in our MD simulations 

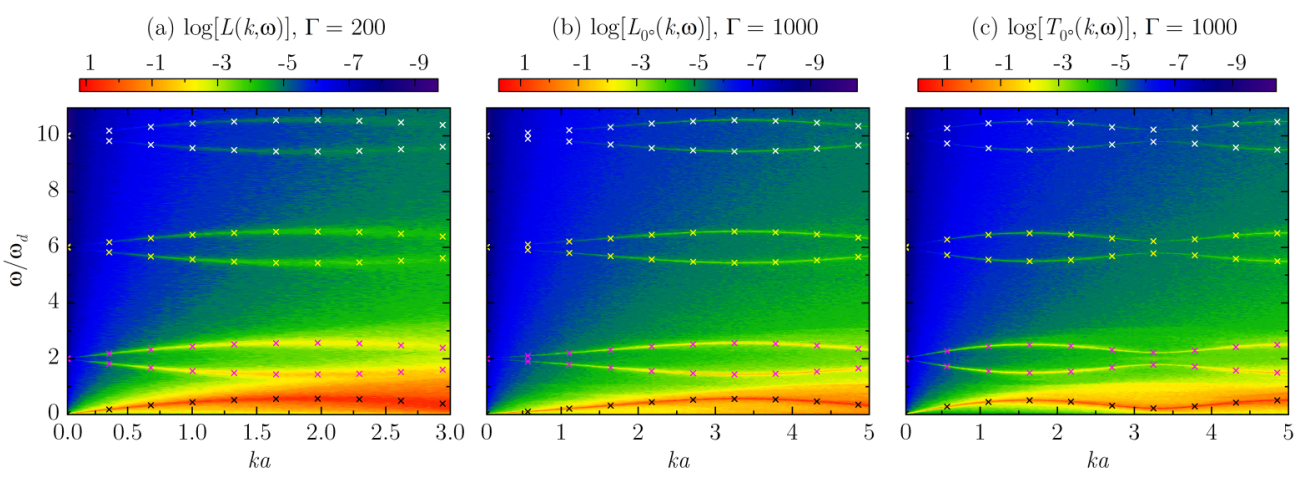

FIG. 2. (a) and (b) Higher harmonics in the 2D system of charged microparticles emerge in the spectrum of the longitudinal current fluctuations for liquid $(\Gamma=200)$ and crystalline states $(\Gamma=1000)$, respectively. (c) The spectrum of the transverse current fluctuations in the crystalline state with $\Gamma=1000$. Crosses correspond to the results of Eq. (6).

that can be varied independently from the computed discharge parameters. One of them is the coupling parameter of the dust particles $\Gamma=Q_{d}^{2} / a k_{B} T_{d}$, where $a=\left(\pi n_{d}\right)^{-1 / 2}$ denotes the Wigner-Seitz radius in $2 \mathrm{D}, Q_{d}$ denotes the dust particle charge, $T_{d}$ denotes the characteristic dust particle temperature, and $k_{B}$ denotes the Boltzmann constant. Although $Q_{d}$ is defined by the plasma parameters of the discharge, the density of dust particles $n_{d}$ can be controlled in experiments as dust particles are immersed into a discharge from the outside. The other one is the so-called screening parameter $\kappa=a / \lambda_{D}$. While the Debye screening length $\lambda_{D}$ is defined by the discharge parameters at the dust particle levitation height, $a$ can be controlled to a certain degree externally.

It is well known from experiments that dust particles form either liquid or crystal states at the considered discharge parameters of $Q_{d} \sim 10^{4}$, where $\kappa$ lies in the range from 1 to 2 and $T_{d}$ is defined by the self-organization process. We note that in our PIC-MCC simulations of the RF discharge, neither the levitation height nor the charge of the dust particles varies upon switching on and off the modulation of the external RF voltage. Nevertheless, $\lambda_{D}$ varies upon switching between the two regimes. Therefore, at a given $n_{d}, \Gamma$ remains constant, and the screening parameter $\kappa$ becomes time dependent. At the considered discharge and external field parameters, $\lambda_{D}$ changes by a factor of 1.43 . Therefore, in our MD simulations, the screening parameter alternates between $\kappa$ and $\kappa / 1.43$, with the standard harmonic RF voltage set to $\kappa=2$. The latter value is characteristic of experiments at the considered parameters of the RF discharge. The temperature of dust particles $T_{d}$ is defined by the self-organization process and is hard to control explicitly. Therefore, taking into account a large value of the microparticle charge, $Q_{d} \simeq 14000 e$ ( $e$ denoting the electron charge), we consider those characteristic $\Gamma$ values of liquid and crystalline states that are observed in experiments of the RF discharge [25].

The MD simulations were performed for $N=10^{4}$ particles of the same mass and charge confined in a square of length $L=\sqrt{\pi N} a$. To eliminate boundary effects, we use periodic boundary conditions. The interaction between particles is described by the screened Coulomb potential

$$
V(r)=\frac{Q^{2} \exp \left(-r / \lambda_{D}\right)}{4 \pi \epsilon_{0} r},
$$

where $r$ denotes the interparticle separation distance. The parameters of $\mathrm{MD}$ simulations were computed using data from the PIC-MCC simulations of the RF discharge as discussed above, and the coordinates $\mathbf{r}_{j}$ of the particles are found by solving the Langevin equation. The presented MD results are computed using the time-dependent screening parameter $\kappa(t)=1.7+0.3 \operatorname{sgn}[\cos (\Omega t)]$, where $\operatorname{sgn}$ is the sign function.

Our central result is the vibrational spectrum (Fig. 2). In order to analyze the natural vibration modes, we compute the spectrum of the longitudinal $L(k, \omega)$ and transverse $T(k, \omega)$ vibrations. Both are defined as the Fourier components of the current density operator $\mathbf{j}(k, t)=\sum_{j=1}^{N} \dot{\mathbf{r}}_{j} \exp \left[i \mathbf{k} \cdot \mathbf{r}_{j}(t)\right]$ with $\dot{\mathbf{r}}_{j}=d \mathbf{r}_{j} / d t$ and $N$ denoting the total number of dust particles $[4,26]$.

The equations for longitudinal and transverse collective excitation spectra read [26]

$$
\begin{aligned}
& L(\mathbf{k}, \omega)=\frac{1}{2 \pi N t_{\text {meas }}}\left|\mathcal{F}_{t}[\lambda(\mathbf{k}, t)]\right|^{2}, \\
& T(\mathbf{k}, \omega)=\frac{1}{2 \pi N t_{\text {meas }}}\left|\mathcal{F}_{t}[\tau(\mathbf{k}, t)]\right|^{2},
\end{aligned}
$$

where $t_{\text {meas }}$ is the measured time and $\mathcal{F}_{t}$ is the temporal Fourier transform. The longitudinal $\lambda(\mathbf{k}, t)$ and transverse $\tau(\mathbf{k}, t)$ components of the microscopic current $\mathbf{j}(\mathbf{k}, t)=$ $\sum_{j=1}^{N} \mathbf{v}_{j}(t) \exp \left[i \mathbf{k} \cdot \mathbf{r}_{j}(t)\right]$ are defined as

$$
\begin{aligned}
\lambda(\mathbf{k}, t) & =\sum_{j=1}^{N} v_{j, x}(t) \exp \left[i \mathbf{k} \mathbf{r}_{j}(t)\right] \\
\tau(\mathbf{k}, t) & =\sum_{j=1}^{N} v_{j, y}(t) \exp \left[i \mathbf{k} \mathbf{r}_{j}(t)\right]
\end{aligned}
$$

where $v_{j, x}(t), v_{j, y}(t)$, and $\mathbf{r}_{j}(t)$ are computed in the MD simulation for each particle $j$ at time $t$. Here, $v_{j, x}(t)$ denotes the velocity in the $x$ direction, and $v_{j, y}(t)$ denotes the velocity in the $y$ direction. In the following, the direction of wave vector $\mathbf{k}$ has been chosen parallel to the $x$ axis and will therefore be denoted by $k$. The results for $L(k, \omega)$ and $T(k, \omega)$ were obtained by averaging over ten simulation runs. 

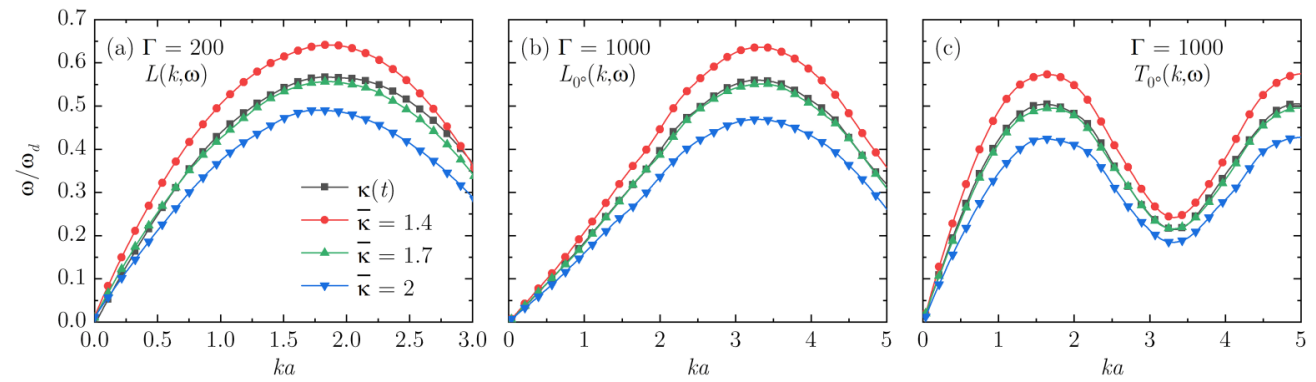

FIG. 3. Acoustic dispersion relations for constant and alternating screening parameters $\kappa$. (a) and (b) The dispersion of longitudinal fluctuations in the liquid and crystalline states, respectively. (c) The dispersion of transverse fluctuations in the crystalline state.

\section{RESULTS}

As illustrated in the dispersion curves in Fig. 2 for $\Omega=$ $2 \omega_{d}$, we have identified the first six modes beyond the standard acoustic mode (at the lowest frequency) of the 2D layered charged particles from the spectrum of their current fluctuations. In Fig. 2(a), we show the longitudinal current fluctuation spectrum in the liquid state at $\Gamma=200$. We note that in this regime, the transverse oscillations are strongly damped. Furthermore, we illustrate the longitudinal and transverse current fluctuation spectra for the crystalline state at $\Gamma=1000$ in Figs. 2(b) and 2(c), respectively. We also infer from Fig. 2 that higher harmonics appear at values of odd multiples of $\Omega$ and that they are split into two branches at finite wave number. Furthermore, the higher harmonics exhibit a gap in reciprocal space, implying that the waves gradually disappear below a certain wave number [27]. For the first pair of higher harmonics, this gap is about $0.1 a^{-1}$ and increases up to about $a^{-1}$ for the second and third pairs of harmonics.

Next, let us analyze the acoustic mode dispersion $\omega[k ; \kappa(t)]$ when the screening parameter $\kappa(t)$ is time dependent. To this end, consider Fig. 3, where we compare the dispersion of the acoustic modes when the screening parameter is fixed against that where it is alternating in time. We have chosen the fixed values $\bar{\kappa}=1.4, \bar{\kappa}=1.7$, and $\bar{\kappa}=2.0$, which correspond to the minimum, mean, and maximum values of the alternating screening parameter $\kappa(t)$. We deduce from this comparison that the dispersion with an alternating screening length agrees well with that when the screening is fixed to the mean value, $\bar{\kappa}=1.7$. This observation stems from the fact that the structural properties are the same in both screening scenarios. This explanation is further supported by the similarities in the radial distribution function (RDF), which is illustrated in Fig. 4.

Finally, we seek an analytical formula for the mode dispersion. Leaning on the similarity in the RDFs, we can take advantage of existing theories such as the well-tested quasilocalized charge approximation (QLCA) [28], which assumes a fixed screening parameter $\bar{\kappa}$ to derive properties of $\omega[k ; \kappa(t)]$. Noting that the acoustic mode resembles the higher harmonics in Fig. 2, we derive the following phenomenological expression:

$$
\omega^{ \pm(j)}(k)=(2 j+1) \Omega \pm \omega(k ; \bar{\kappa}),
$$

where $j$ is an integer number, $\Omega \neq 0$, and the " + " and "-" signs refer to the upper and lower branches of the modes, which coalesce into a single mode when $k \rightarrow 0$ and $\omega(k \rightarrow 0 ; \bar{\kappa}) \rightarrow 0$. Note that this expression is valid for both longitudinal and transverse modes, where the corresponding $\omega(k ; \bar{\kappa})$ needs to be used.

The prognostic value of Eq. (6) is demonstrated in Fig. 2. The crosses are obtained from evaluating this analytical expression for the first six higher harmonics (i.e., $j<3$ ), which is in full agreement with our simulation results. Note that this expression is valid for both longitudinal and transverse modes. We also note that close above the acoustic mode, i.e., when $\omega^{ \pm(j)}(k)<\omega(k)$, the hybridization of the modes leads to a fast heating and melting of the system [29]. Therefore the condition $\omega^{ \pm(i)}(k)>\omega(k)$ is necessary for the observation of a stable system in both simulation and experiment.

\section{CONCLUSIONS AND OUTLOOK}

Crowned with an expression in Eq. (6) for the mode dispersion, we have predicted the emergence of higher harmonics in a 2D layer of strongly correlated charged microparticles in a medium with alternating screening. Thereby, we pave the way for an experimental observation of this nonlinear

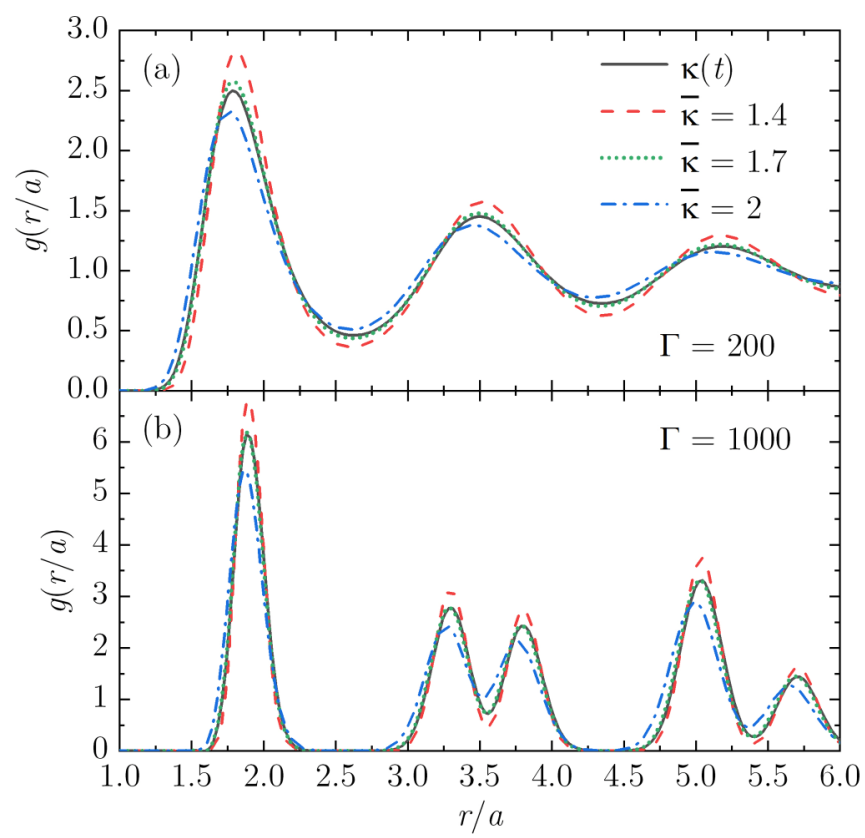

FIG. 4. (a) and (b) Radial distribution functions of charged microparticles for two coupling parameters $\Gamma$ with constant and alternating screening constants $\kappa$. 
phenomenon. We ensure the experimental feasibility of our prediction by assuming realistic plasma parameters in the RF discharge. This was only possible due to the self-consistent PIC-MCC simulations we performed.

Furthermore, we anticipate that the observation of the presented collective excitations could be realized in a 3D system of charged microparticles under microgravity conditions [30], where alternating ambient plasma parameters can be created using an external source providing gas ionization, which is turned on and off periodically.

A peculiar property of the dispersion relation in Eq. (6) is that the group and phase velocities are directed opposite to each other:

$$
\frac{\omega^{-(i)}}{k} \frac{d \omega^{-(i)}}{d k}<0
$$

This phenomenon is known as negative refraction [31,32]. In contrast to that, the dispersion $\omega^{+(i)}(k)$ corresponds to a positively refracting state. In general, a medium with dispersion modes resulting in both negative and positive refraction is called self-conjugate [33]. Therefore we have numerically shown that a 2D layer of charged microparticles with alternating screening is a self-conjugate state with negative refraction.

Let us finally take a look at the presented results from a more general point of view. In crystals, particle oscillations can be described using the harmonic approximation. In our case, the motion of the dust particles is governed by

$$
\ddot{u}_{i}+\sum_{j=1}^{3 N} \bar{\lambda}_{i j}(1+\epsilon \cos (\Omega t)) u_{j}=0,
$$

where $\bar{\lambda}_{i j}$ is a stiffness coefficient defined by the pairinteraction potential among the particles, $\epsilon<1$, and $\mathbf{u}$ is a displacement vector. Equation (8) is the $N$-particle version of Mathieu's differential equation. Mathieu's equation-an archetypal equation in the theory of nonlinear dynamicsspans a wide application range from quantum mechanics to general relativity. Simple plane-wave analysis is not sufficient to find solutions of this equation due to the $\cos (\Omega t)$ term. Clearly, one needs to invoke the theory of nonlinear oscillations. To this end, one can further simplify the problem by considering the equation in 1D. Essentially, as confirmed by our simulations, the observed waves and their dispersion delineated by Eq. (6) remain valid in 1D (see Appendix B). In this case, Eq. (6) reduces to the 1D version of Mathieu's differential equation [34]. However, a direct use of the Mathieu functions (the solutions of Mathieu's equation) does not straightforwardly lead to the dispersion relation even in 1D. However, Eq. (6) can be understood as such a solution obtained from the simulation of the vibrational spectrum. Therefore a correlated many-particle system governed by the $N$-particle Mathieu equation is expected to exhibit higher harmonics as shown in this paper.

In closing, we speculate that our findings can serve as a test bed for studying the fundamental physics of a self-conjugate state with negative refraction in strongly correlated systems on the kinetic level.

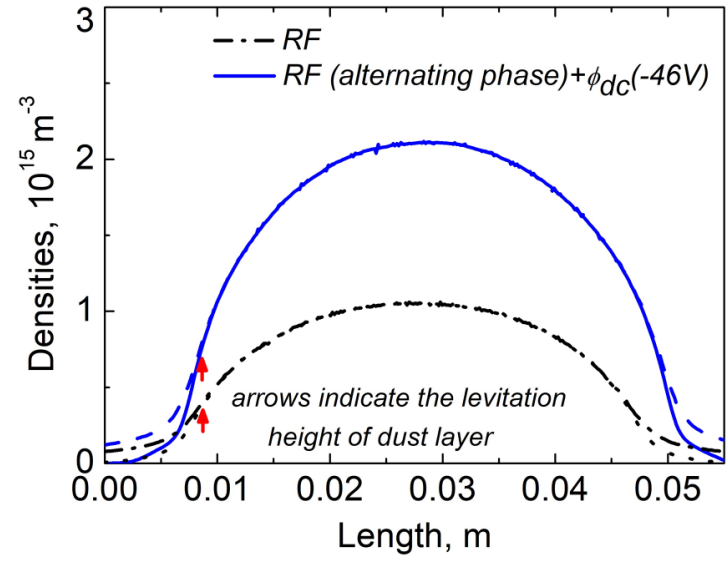

FIG. 5. Spatial profile of the ion (dashed and dash-dotted lines) and electron (solid and dotted lines) densities for the different excitation wave forms.

\section{ACKNOWLEDGMENTS}

N.K.B. thanks Zoltan Donkó for hospitality during N.K.B.'s internship at the Institute for Solid State Physics and Optics within the Wigner Research Center for Physics and for supervising the PIC simulations. This work was partly funded by the Center for Advanced Systems Understanding (CASUS), which is financed by the German Federal Ministry of Education and Research (BMBF) and by the Saxon Ministry for Science, Culture and Tourism (SMWK) with tax funds on the basis of the budget approved by the Saxon State Parliament.

\section{APPENDIX A: SIMULATION DETAILS}

A detailed description of the PIC-MCC simulation code used to model the RF discharge is given in Refs. [22,23].

In Fig. 5, the density profiles of the electrons and ions are shown for different excitation wave forms. In comparison with the standard harmonic RF excitation, the alternating-phase driven voltage leads to an increase in the electron and ion densities by a factor of about 2.7. The additional dc bias results in a decrease in the peak density. In Fig. 6, the spatiotemporal profiles of the electron density and effective temperature from the mean energy of electrons measured in the simulation are shown for a harmonic RF excitation [Figs. 6(a) and 6(c)] and for an alternating-phase excitation with an additional $\mathrm{dc}$ bias [Figs. 6(b) and 6(d)]. The spatial profile of the Debye screening length computed using plasma parameters averaged over the period of the fundamental frequency of the RF discharge $(13.56 \mathrm{MHz})$ is presented in Fig. 7. A discussion of the impact of the alternating-phase excitation and of the impact of the additional dc bias on the spatiotemporal profiles of the electron temperature is given in Ref. [35].

We scanned the grid points between electrodes to find the levitation position of dust particles based on the PIC-MCC simulation result of the RF discharge. Here, we present a brief description of the model used for the calculation of forces which define the levitation position in the RF discharge. Further details of the plasma-dust particle interaction model 

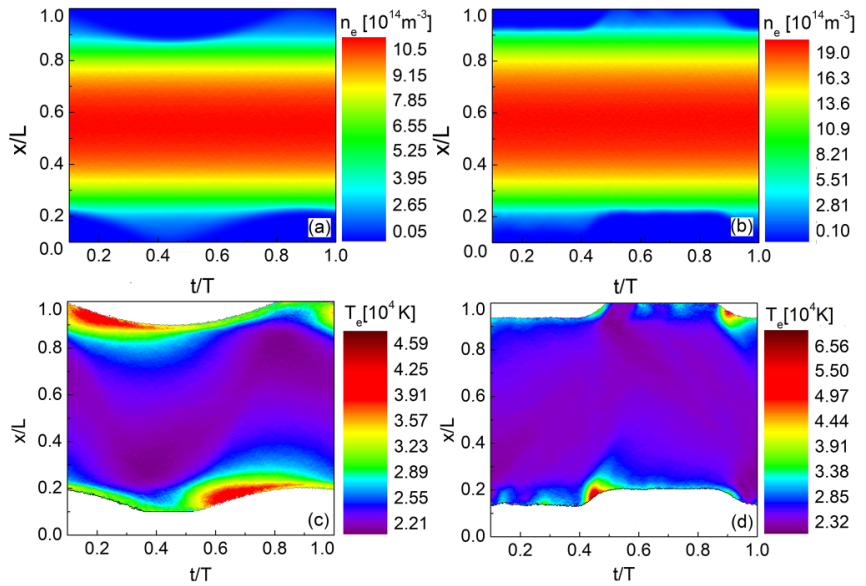

FIG. 6. Spatiotemporal profile of the electron density [(a) and (b)] and the electron temperature [(c) and (d)] in the discharge which results from the applied voltage wave forms; (a) and (c) correspond to the harmonic RF excitation, and (b) and (d) correspond to the alternating-phase excitation with dc bias. The time is given in units of the period $T$ of the fundamental frequency $(13.56 \mathrm{MHz})$.

are given in Ref. [23], where the accuracy of the method was confirmed by comparison with experimental data.

The floating potential $\varphi_{\mathrm{d}}$ is calculated from the electron and ion fluxes in the dust particles on the basis of the orbital motion limited (OML) approximation [36]. At each RF cycle, based on the data from the PIC-MCC simulation, the electron and ion fluxes $\left(\Gamma_{\mathrm{e}}\right.$ and $\left.\Gamma_{\mathrm{i}}\right)$ are calculated using

$$
\Gamma_{\mathrm{e}} \propto \sum_{p} W_{\mathrm{e}} v_{p} n_{d}(x) \sigma_{\mathrm{ed}}\left[\varepsilon_{p}, \varphi_{\mathrm{d}}\left(x_{\mathrm{k}}\right)\right]
$$

and

$$
\Gamma_{\mathrm{i}} \propto \sum_{p} W_{\mathrm{i}} v_{p} n_{d}(x) \sigma_{\mathrm{id}}\left[\varepsilon_{p}, \varphi_{\mathrm{d}}\left(x_{\mathrm{k}}\right)\right],
$$

where $W$ denotes the weight of a superparticle, $v_{p}$ and $\varepsilon_{p}$ denote the velocity and energy of the $p$ th electron or ion, $n_{d}$ denotes the dust density, and $\sigma_{\text {ed }}$ and $\sigma_{\text {id }}$ denote the electrondust and ion-dust collision ("collection") cross sections [37].

The floating potential of the dust particles is found iteratively by enforcing the requirement that (A1) and (A2) become equal in the stationary state. Having obtained the

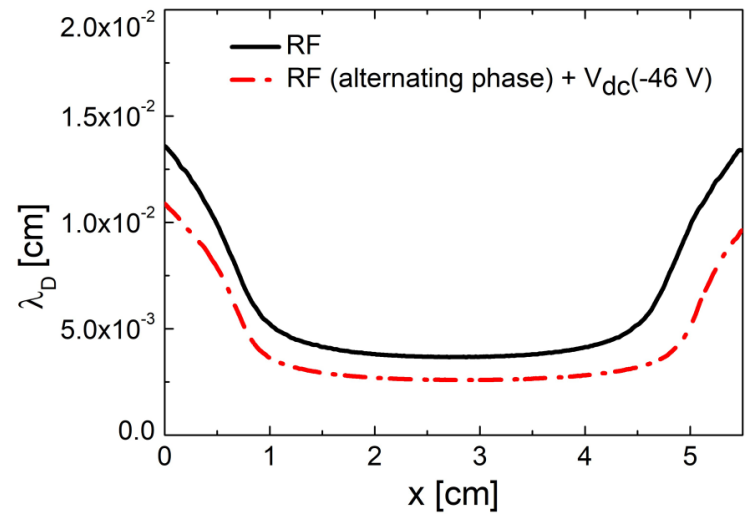

FIG. 7. Spatial profile of the Debye screening length.

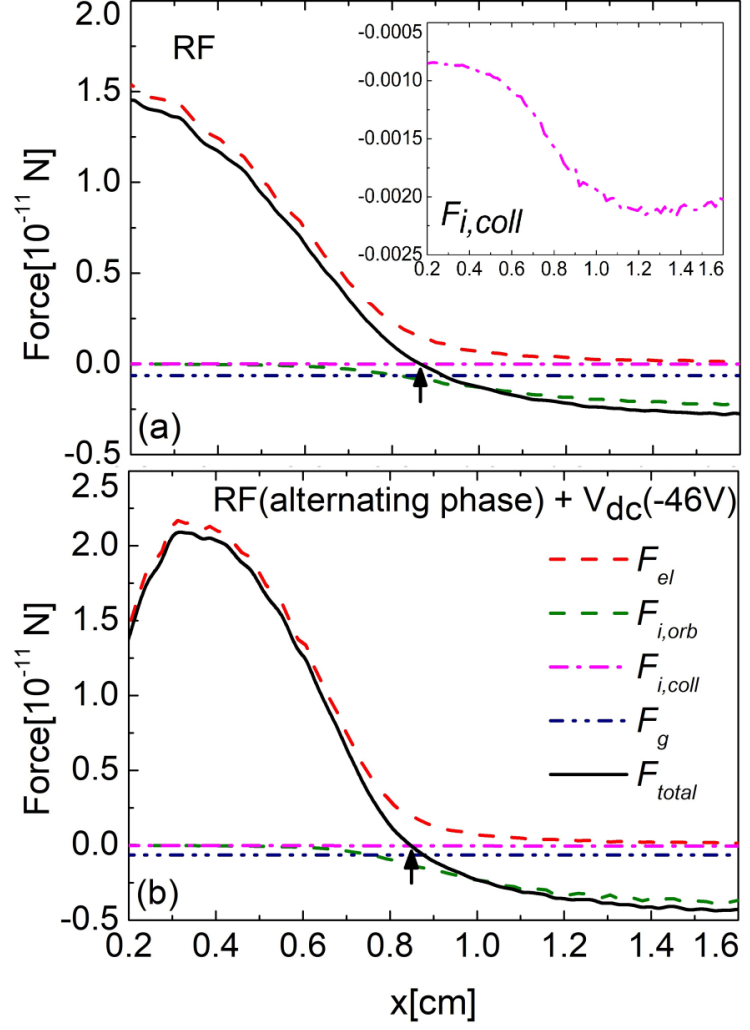

FIG. 8. Individual force components and the total force acting on the microparticle for the different excitation wave forms considered. Arrows indicate the equilibrium microparticle levitation position defined by $F_{\text {tot }}=0$. (a) Harmonic RF excitation, where at the levitation position $n_{e}=3.9 \times 10^{14} \mathrm{~m}^{-3}, n_{i}=3.72 \times 10^{14} \mathrm{~m}^{-3}, \lambda_{D}=6.153 \times$ $10^{-5} \mathrm{~m}, Q_{d}=13990 e$, and $x_{d}=0.0086 \mathrm{~m}$. (b) Alternating-phase excitation with $\mathrm{dc}$ bias, where at the levitation position $n_{e}=$ $7.528 \times 10^{14} \mathrm{~m}^{-3}, n_{i}=7.213 \times 10_{14} \mathrm{~m}^{-3}, \lambda_{D}=4.361 \times 10^{-5} \mathrm{~m}$, $Q_{d}=13692 e$, and $x_{d}=0.0085 \mathrm{~m}$.

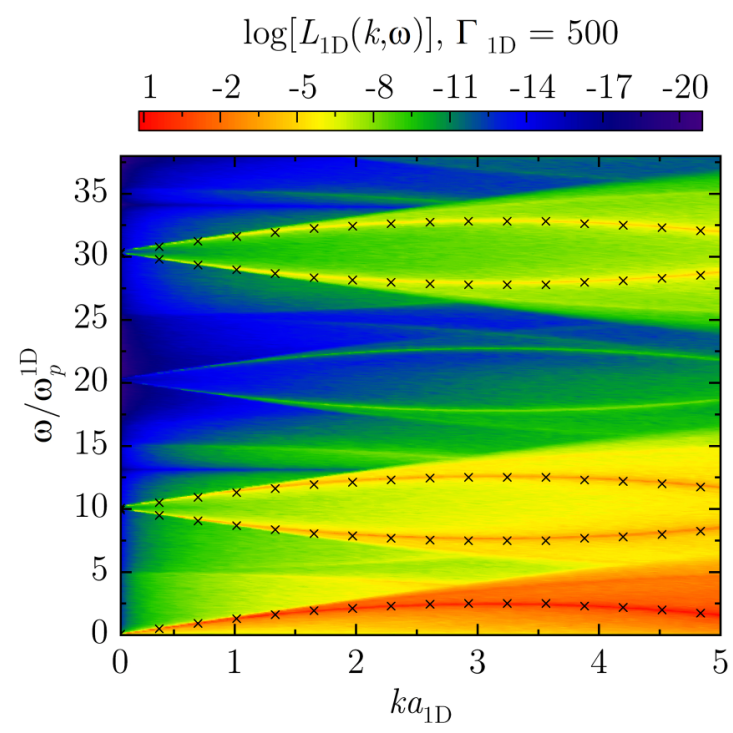

FIG. 9. Higher harmonics emerge in the dispersion relation of a chain of charged microparticles. 
floating potential at a given grid point, the equilibrium charge of the dust particle is calculated as

$$
Q_{\mathrm{d}}=4 \pi \varepsilon_{0} r_{d} \varphi_{\mathrm{d}}\left(x_{\mathrm{k}}\right),
$$

where $\varepsilon_{0}$ denotes the dielectric constant, $\varphi_{\mathrm{d}}$ is the floating potential, and $r_{d}$ is the dust particle radius.

The equilibrium levitation position of the dust particle is derived from the force-balance equation

$$
F_{\text {tot }}=F_{\mathrm{el}}\left(x_{\mathrm{d}}\right)-F_{\mathrm{g}}-F_{\mathrm{i}}\left(x_{\mathrm{d}}\right)=0,
$$

where $F_{\text {el }}$ denotes electrostatic force, $F_{\mathrm{g}}$ denotes the gravitational force, and $F_{\mathrm{i}}$ denotes the ion-drag force. The ion-drag force $F_{\mathrm{i}}(x)=F_{\mathrm{i} \text {, coll }}(x)+F_{\mathrm{i} \text {,orb }}(x)$ consists of two components: (i) $F_{\mathrm{i}, \text { coll }}(x)$ is due to ions absorbed by the dust particle (collection force), and (ii) $F_{\mathrm{i}, \text { orb }}(x)$ is due to ions deflected by the charged dust particle (orbit force). Further details of the used models and approximations for different components of the total force are given in Refs. [23,35].
In Fig. 8, the individual force components and the total force acting on a dust particle are shown. In Fig. 8(a) the standard harmonic RF voltage is applied, whereas in Fig. 8(b) an alternating voltage with an additional dc voltage is applied.

\section{APPENDIX B: 1D SYSTEM WITH ALTERNATING SCREENING}

Figure 9 illustrates the spectrum of the current fluctuations in 1D. The MD simulation of the 1D system was performed using $N=1000$ particles, where periodic boundary conditions were imposed. The time-dependent screening parameter takes values between $\kappa=1.4$ and $\kappa=2$ with a frequency $\Omega=10 \omega_{d}$. In Fig. 9, the crosses indicate the results obtained from the analytical dispersion presented in this paper. We note that a 1D chain of the charged microparticles inside a glass box is investigated in dusty plasma experiments $[38,39]$.
[1] M. Lemeshko, R. V. Krems, J. M. Doyle, and S. Kais, Manipulation of molecules with electromagnetic fields, Mol. Phys. 111, 1648 (2013).

[2] S. A. Khrapak, N. P. Kryuchkov, and S. O. Yurchenko, Thermodynamics and dynamics of two-dimensional systems with dipolelike repulsive interactions, Phys. Rev. E 97, 022616 (2018).

[3] P. Hartmann, Z. Donkó, T. Ott, H. Kählert, and M. Bonitz, Magnetoplasmons in Rotating Dusty Plasmas, Phys. Rev. Lett. 111, 155002 (2013).

[4] T. Ott, H. Kählert, A. Reynolds, and M. Bonitz, Oscillation Spectrum of a Magnetized Strongly Coupled One-Component Plasma, Phys. Rev. Lett. 108, 255002 (2012).

[5] X.-J. Chen, Exploring high-temperature superconductivity in hard matter close to structural instability, Matter Radiat. Extremes 5, 068102 (2020).

[6] S. Imai, A. Ono, and S. Ishihara, High Harmonic Generation in a Correlated Electron System, Phys. Rev. Lett. 124, 157404 (2020).

[7] B. Wang, J. A. Rodríguez, O. Miller, and M. A. Cappelli, Reconfigurable plasma-dielectric hybrid photonic crystal as a platform for electromagnetic wave manipulation and computing, Phys. Plasmas 28, 043502 (2021).

[8] D. Grošelj, C. H. K. Chen, A. Mallet, R. Samtaney, K. Schneider, and F. Jenko, Kinetic Turbulence in Astrophysical Plasmas: Waves and/or Structures? Phys. Rev. X 9, 031037 (2019).

[9] S. Sundar and Z. A. Moldabekov, Oblique magnetic field influence on the wakefield in complex plasmas, Plasma Phys. Controlled Fusion 62, 105018 (2020).

[10] N. P. Kryuchkov, L. A. Mistryukova, A. V. Sapelkin, V. V. Brazhkin, and S. O. Yurchenko, Universal Effect of Excitation Dispersion on the Heat Capacity and Gapped States in Fluids, Phys. Rev. Lett. 125, 125501 (2020).

[11] T. Dornheim, S. Groth, J. Vorberger, and M. Bonitz, Ab Initio Path Integral Monte Carlo Results for the Dynamic Structure Factor of Correlated Electrons: From the Electron Liquid to Warm Dense Matter, Phys. Rev. Lett. 121, 255001 (2018).
[12] Y. Takada, Emergence of an excitonic collective mode in the dilute electron gas, Phys. Rev. B 94, 245106 (2016).

[13] J. Bardeen, L. N. Cooper, and J. R. Schrieffer, Microscopic theory of superconductivity, Phys. Rev. 106, 162 (1957).

[14] C. C. Chien, S. Peotta, and M. Di Ventra, Quantum transport in ultracold atoms, Nat. Phys. 11, 998 (2015).

[15] G. E. Morfill and A. V. Ivlev, Complex plasmas: An interdisciplinary research field, Rev. Mod. Phys. 81, 1353 (2009).

[16] R. E. Boltnev, M. M. Vasiliev, E. A. Kononov, and O. F. Petrov, Formation of solid helical filaments at temperatures of superfluid helium as self-organization phenomena in ultracold dusty plasma, Sci. Rep. 9, 3261 (2019)

[17] S. Sundar and Z. A. Moldabekov, Ultracold ions wake in dusty plasmas, New J. Phys. 22, 033028 (2020).

[18] S. Sundar and Z. A. Moldabekov, Plasma-grain interaction in ultracold complex plasmas, Phys. Plasmas 27, 033701 (2020).

[19] P. Hartmann, A. Zs. Kovács, A. M. Douglass, J. C. Reyes, L. S. Matthews, and T. W. Hyde, Slow Plastic Creep of 2D Dusty Plasma Solids, Phys. Rev. Lett. 113, 025002 (2014).

[20] Z. Haralson and J. Goree, Overestimation of Viscosity by the Green-Kubo Method in a Dusty Plasma Experiment, Phys. Rev. Lett. 118, 195001 (2017).

[21] A. V. Ivlev, J. Bartnick, M. Heinen, C.-R. Du, V. Nosenko, and H. Löwen, Statistical Mechanics Where Newton's Third Law Is Broken, Phys. Rev. X 5, 011035 (2015).

[22] Z. Donkó, Particle simulation methods for studies of lowpressure plasma sources, Plasma Sources Sci. Technol. 20, 024001 (2011).

[23] N. Kh. Bastykova, A. Zs. Kovács, I. Korolov, S. K. Kodanova, T. S. Ramazanov, P. Hartmann, and Z. Donkó, Controlled levitation of dust particles in RF+DC discharges, Contrib. Plasma Phys. 55, 671 (2015).

[24] G. J. M. Hagelaar, Z. Donko, and N. Dyatko, Modification of the Coulomb Logarithm due to Electron-Neutral Collisions, Phys. Rev. Lett. 123, 025004 (2019).

[25] M. Bonitz, C. Henning, and D. Block, Complex plasmas: a laboratory for strong correlations, Rep. Prog. Phys. 73, 066501 (2010). 
[26] J. P. Hansen, I. R. McDonald, and E. L. Pollock, Statistical mechanics of dense ionized matter. III. Dynamical properties of the classical one-component plasma, Phys. Rev. A 11, 1025 (1975).

[27] N. P. Kryuchkov, L. A. Mistryukova, V. V. Brazhkin, and S. O. Yurchenko, Excitation spectra in fluids: How to analyze them properly, Sci. Rep. 9, 10483 (2019).

[28] G. J. Kalman, P. Hartmann, Z. Donkó, and M. Rosenberg, TwoDimensional Yukawa Liquids: Correlation and Dynamics, Phys. Rev. Lett. 92, 065001 (2004).

[29] L. Couëdel, V. Nosenko, S. Zhdanov, A. V. Ivlev, I. Laut, E. V. Yakovlev, N. P. Kryuchkov, P. V. Ovcharov, A. M. Lipaev, and S. O. Yurchenko, Experimental studies of two-dimensional complex plasma crystals: waves and instabilities, Usp. Fiz. Nauk 62, 1000 (2019).

[30] C. A. Knapek, U. Konopka, D. P. Mohr, P. Huber, A. M. Lipaev, and H. M. Thomas, Zyflex: Next generation plasma chamber for complex plasma research in space, Rev. Sci. Instrum. 92, 103505 (2021).

[31] J. B. Pendry, A chiral route to negative refraction, Science 306, 1353 (2004).

[32] V. V. Cheianov, V. Fal'ko, and B. L. Altshuler, The focusing of electron flow and a Veselago lens in graphene $p-n$ junctions, Science 315, 1252 (2007).

[33] J. B. Pendry, Time reversal and negative refraction, Science 322, 71 (2008).

[34] E. Mathieu, Mémoire sur le mouvement vibratoire d'une membrane de forme elliptique, J. Math. Pures Appl. 2, 137 (1868).

[35] N. Kh. Bastykova, Z. Donkó, S. K. Kodanova, T. S. Ramazanov, and Zh. A. Moldabekov, Manipulation of dusty plasma properties via driving voltage waveform tailoring in a capacitive radiofrequency discharge, IEEE Trans. Plasma Sci. 44, 545 (2016).

[36] A. L. Alexandrov, I. V. Schweigert, and F. M. Peeters, A nonMaxwellian kinetic approach for charging of dust particles in discharge plasmas, New J. Phys. 10, 093025 (2008).

[37] A. Khrapak and A. V. Ivlev, Basic plasma-particle interactions, in Complex and Dusty Plasmas, edited by V. E. Fortov and G. E. Morfill (CRC, Boca Raton, 2010), p. 104.

[38] J. Kong, K. Qiao, L. S. Matthews, and T. W. Hyde, Interaction force in a vertical dust chain inside a glass box, Phys. Rev. E 90, 013107 (2014)

[39] L. Scott, N. Ellis, M. Chen, L. S. Matthews, and T. W. Hyde, Mapping the plasma potential in a glass box, IEEE Trans. Plasma Sci. 47, 3079 (2019). 